\title{
Bordetella hinzii Endocarditis, A Clinical Case Not Previously Described
}

\author{
María Mateos González ${ }^{1}$, Maria Pilar Cubo Romanoํㅜ, Maria Teresa de Guzmán García Monge ${ }^{1}$, \\ Beatriz Berzal Martín², Aída Sánchez García ${ }^{3}$ \\ ${ }^{1}$ Department of Internal Medicine, Infanta Cristina University Hospital, Parla, Madrid \\ ${ }^{2}$ Department of Cardiology, Infanta Cristina University Hospital, Parla, Madrid \\ ${ }^{3}$ Central Laboratory, BR Salud, Infanta Sofía Hospital, San Sebastián de los Reyes, Madrid
}

\section{Doi: 10.12890/2019_000994 - European Journal of Case Reports in Internal Medicine - ๑ EFIM 2019}

Received: $13 / 11 / 2018$

Accepted: $17 / 12 / 2018$

Published: 04/02/2019

\begin{abstract}
How to cite this article: Mateos González M, Cubo Romano MP, de Guzmán García Monge MT, Berzal Martín B, Sánchez García A. Bordetella hinzii
\end{abstract} endocarditis, a clinical case not previously described. EJCRIM 2019;6: doi:10.12890/2019_000994.

Conflicts of Interests: The Authors declare that there are no competing interests.

This article is licensed under a Commons Attribution Non-Commercial 4.0 License

\section{ABSTRACT}

Objective: To review infections by Bordetella hinzii.

Materials and methods: A 79-year-old male patient, with a chronic aortic valve biological prosthesis, presented to hospital because of fever. First examinations were normal. However, 72 hours later B. hinzii was isolated in blood cultures, and so meropenem was prescribed. Nevertheless, fever and B. hinzii bacteraemia were still present 7 days later.

Results: The transoesophageal echocardiogram revealed an enlarged image suggesting a periprosthetic abscess, confirmed with a PET-CT scan. The patient was sent for cardiac surgery, and biopsy samples confirmed the presence of $B$. hinzii.

Conclusion: There are very few cases of $B$. hinzii infection in humans. Ours is the first described case of $B$. hinzii endocarditis.

\section{LEARNING POINTS}

- Bordetella hinzii is commonly detected in poultry but very few cases have been described in humans since it was first isolated in 1994 . Some type of immunosuppression is identified in $90 \%$ of patients.

- B. hinzii is frequently resistant to many antibiotics including $\beta$-lactams, macrolides, quinolones and cephalosporins. The diagnosis is often difficult using conventional phenotypic methods, so genotypic methods may be necessary for confirmation.

- Ours is the first described case of infection by B. hinzii with endocardial-vascular involvement. However, cases of endocarditis due to other Bordetella species such as B. holmesii have been documented.

\section{KEYWORDS}

Bordetella hinzii, endocarditis, valvular prosthesis

\section{INTRODUCTION}

Human infection caused by Bordetella hinzii is very uncommon. The main risk factor is immunosuppression, and infection has so far presented as bacteraemia, respiratory symptoms or cholangitis. We present the case of a patient with acute endocarditis caused by $B$. hinzii infection of an aortic prosthetic valve. 


\section{CASE DESCRIPTION}

In February 2018, a 79-year-old male patient was admitted to the emergency room of Hospital Infanta Cristina, Madrid, Spain because of fever, cough and yellow expectoration, accompanied by alterations in behaviour, dysarthria and unsteady gait. The patient had undergone surgery in 2013 for severe symptomatic aortic valve stenosis, and had an aortic valve biological prosthesis inserted. He had chronic liver disease (class B on the Child Pugh score, with portal hypertension), chronic kidney disease stage III, well-controlled diabetes mellitus type II (HbA1c 5.2\%) and high blood pressure. He also had colon adenocarcinoma in remission after right hemicolectomy in 2000. On admission, initial tests showed an increase in C-reactive protein, thrombocytopenia, hyperbilirubinemia and mild hypertransaminasemia. The cranial CT scan, chest x-ray and abdominal ultrasound were normal. Urine, sputum and blood cultures were drawn and antibiotic treatment with ceftriaxone was prescribed. The urine and sputum cultures were negative.

A Gram-negative bacillus was isolated in blood cultures 72 hours later and identified as Bordetella hinzii using matrix-assisted laser desorption ionization-time of flight mass spectrometry (MALDI-TOF MS; Bruker, Germany). In vitro antibacterial susceptibilities were evaluated using the gradient (E-test; bioMerieux, France) strip method. MICs were: cefotaxime, <256 mg/l; imipenem, 2 mg/l; levofloxacin, 1 $\mathrm{mg} / \mathrm{l}$; erythromycin, $16 \mathrm{mg} / \mathrm{l}$; minocycline, $1 \mathrm{mg} / \mathrm{l}$; and co-trimoxazole, $0.1 \mathrm{mg} / \mathrm{l}$. The identification was confirmed using molecular techniques based on $16 \mathrm{~S}$ rDNA sequencing.

Antibiotic therapy was scaled up to meropenem, improving the respiratory and neurological symptoms. However, 7 days later, fever was still present, and persistent bacteraemia due to B. hinzii was detected in a new batch of blood cultures. It was therefore decided to include piperacillin-tazobactam and to expand the study: a chest-abdomen-pelvis CT scan did not show any relevant pathological findings, but a transoesophageal echocardiogram revealed an enlarged image $(29 \times 14 \mathrm{~mm})$ in the anterior segment of the aortic prosthesis, which continued in the initial segment of the ascending aorta, suggesting a periaortic abscess within the preserved aortic valve (Fig. 1). A PET-CT scan showed an increase in the pathological metabolism of the periaortic valve prosthesis, consistent with a perivascular infectious pathology (Fig. 2). Given the diagnosis of infective endocarditis caused by B. hinzii in the periaortic prosthetic valve, the patient was sent to the cardiac surgery service of our referral hospital where the prosthetic aortic valve and mitral valve were replaced, and the interatrial septum (where the abscess was observed) resected. Samples of the periprosthetic abscess collected during the operation confirmed the presence of $B$. hinzii. The final diagnosis was acute endocarditis caused by $B$. hinzii of the aortic prosthetic valve, a continuing mitral abscess and a septal abscess.

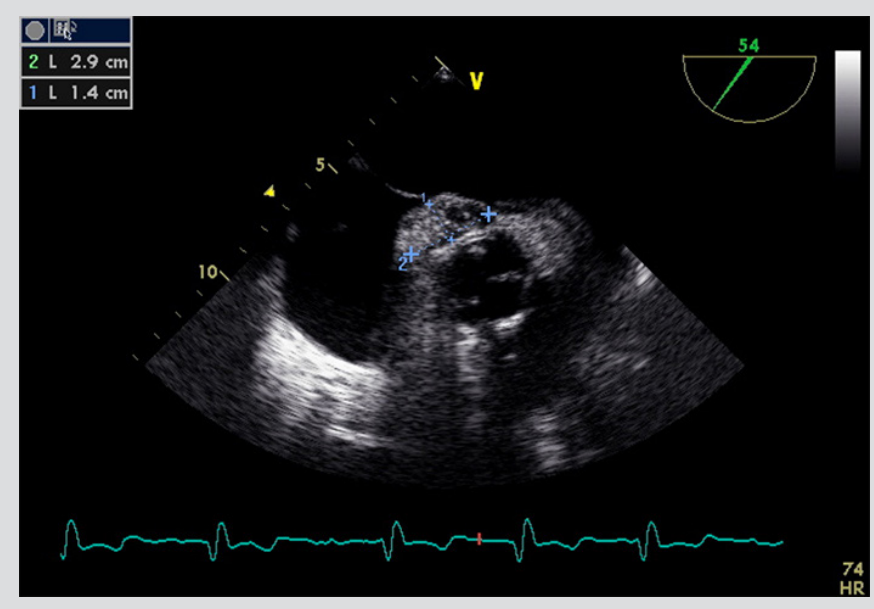

Figure 1. A transesophageal echocardiogram revealed an hyperechoic enlarged image measuring $2.9 \mathrm{~cm}$ long and $1.4 \mathrm{~cm}$ wide.

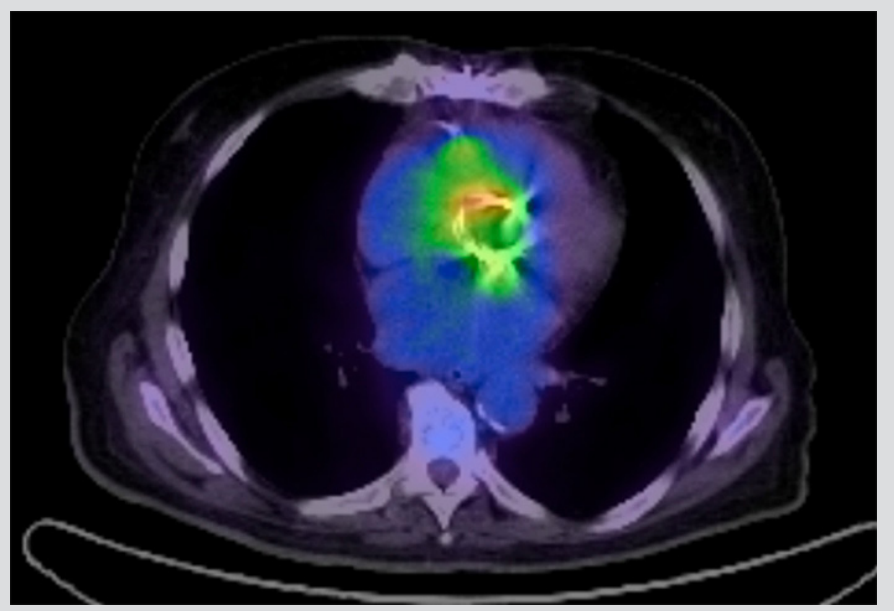

Figure 2. A PET/TC scan showed a pathological metabolism of the periaortic valve prosthesis, consistent with a perivascular infectious pathology.

\section{DISCUSSION}

B. hinzii is a Gram-negative bacillus commonly detected in poultry. It was isolated in a patient with AIDS for the first time in 1994. We have found only 10 cases of symptomatic patients since described in the literature.

B. hinzii is considered to be an emerging opportunistic pathogen. In $90 \%$ of cases, some type of immunosuppression is identified (AIDS, solid organ transplant, bone marrow transplant, cystic fibrosis, myelodysplastic syndrome, B lymphoma, acute myeloid leukaemia, and carcinoma of the larynx). In five of the described cases, B. hinzii was isolated in sputum, in four cases in blood cultures, and in one case in bile. Only one 
of the cases showed contact with poultry ${ }^{[1]}$. Isolation in sputum and bile was frequently associated with other bacteria such as Nocardia ${ }^{[2]}$, MRSA, Staphylococcus epidermidis or Klebsiella. A few cases have been described in immunocompetent patients, as well as in respiratory samples taken from asymptomatic patients. The diagnosis is often difficult using conventional phenotypic methods, and genotypic methods for confirmation may be necessary ${ }^{[2,3]}$.

B. hinzii is frequently resistant to many antibiotics including $\beta$-lactams, macrolides, quinolones and cephalosporins ${ }^{[1]}$.

Ours is the first described B. hinzii infection with endocardial-vascular involvement. There are documented cases of endocarditis due to other Bordetella species such as B. holmesii ${ }^{[4]}$. No contact with poultry was documented in our case. Possible predisposing factors for infection in our patient were cirrhosis, kidney disease and diabetes. In addition, the prosthetic aortic valve was a risk factor for endocarditis in our patient, but no other risk factor was found that could help us identify where or how the patient was infected.

\section{REFERENCES}

1. Fabre A, Dupin C, Bénézit F, Goret J, Piau C, Jouneau S, et al. Opportunistic pulmonary Bordetella hinzii infection after avian exposure. Emerg Infect Dis 2015;21.

2. Pittet LF, Emonet S, Schrenzel J, Siegrist CA, Posfay-Barbe KM. Bordetella holmesii: an under-recognised Bordetella species. Lancet Infect Dis 2014;14:510-519.

3. Hristova AC, Auwaerterb PG, Romagnolia M, Carrolla KC. Bordetella hinzii septicemia in association with Epstein-Barr virus viremia and an Epstein-Barr virus-associated diffuse large B-cell lymphoma. Diagn Microbiol Infect Dis 2008;61:484-486.

4. Gadea I, Cuenca-Estrella M, Benito N, Blanco A, Fernández-Guerrero ML, Valero-Guillén PL, Soriano F. Bordetella hinzii, a "new" opportunistic pathogen to think about. J Infect 2000;40:298-299. 